\title{
Publisher Correction: Restoration of visual function in adult mice with an inherited retinal disease via adenine base editing
}

Susie Suh (D), Elliot H. Choi, Henri Leinonen (D), Andrzej T. Foik (D), Gregory A. Newby, Wei-Hsi Yeh (D), Zhiqian Dong, Philip D. Kiser (D), David C. Lyon (D), David R. Liu (D) and Krzysztof Palczewski (D)

Correction to: Nature Biomedical Engineering https://doi.org/10.1038/s41551-020-00632-6, published online 19 October 2020.

In the version of this Article originally published, in the second sentence of the Abstract, 'adeno-associated virus' should have been replaced with 'lentivirus'; that is, the sentence should have read 'Here, we show, in adult mice, that a subretinal injection of a lentivirus expressing an $\mathrm{ABE}$... This has now been corrected.

Published online: 29 October 2020

https://doi.org/10.1038/s41551-020-00652-2

(c) The Author(s), under exclusive licence to Springer Nature Limited 2020 